\title{
ISOSPECTRAL FAMILIES OF CONFORMALLY EQUIVALENT RIEMANNIAN METRICS
}

\author{
ROBERT BROOKS AND CAROLYN GORDON
}

Two Riemannian manifolds are said to be isospectral if their associated Laplace operators have the same spectrum. Many examples of pairs and of continuous families of isospectral manifolds have appeared in recent years; see [Ber] for a discussion. The first examples of pairs of conformally equivalent isospectral manifolds appeared in [BT], and a general construction was given by Brooks, Perry, and Yang [BPY]. The purpose of this note is to construct continuous families of isospectral conformally equivalent manifolds, using the method of [BPY].

We begin with an example:

Let $F$ be the Lie group of all $7 \times 7$ matrices of the form

$$
\left(\begin{array}{ccccccc}
1 & x_{1} & x_{2} & z_{1} & & & \\
0 & 1 & 0 & y_{1} & & & \\
0 & 0 & 1 & y_{2} & & & \\
0 & 0 & 0 & 1 & & & \\
& & & & 1 & x_{0}+x_{1} & z_{2} \\
& & & & 0 & 1 & y_{2} \\
& & & & 0 & 0 & 1
\end{array}\right) .
$$

We denote elements of $F$ by

$$
f=f\left(x_{0}, x_{1}, x_{2}, y_{1}, y_{2}, z_{1}, z_{2}\right) .
$$

Let $\Gamma$ be the integer lattice in $F$, that is, the set of $f$ 's where $\left(x_{0}, x_{1}, x_{2}, y_{1}, y_{2}, z_{1}, z_{2}\right) \in \mathbb{Z}$, and let $g$ be any left-invariant metric on $F$. Let $v$ be any smooth nonconstant periodic function on $\mathbb{R}$ of period 1 , and define, for each $t, u_{t} \in C^{\infty}(F)$ by the formula

$$
u_{t}(f)=v\left(x_{0}+t\right) .
$$

Received by the editors July 26, 1989 .

1980 Mathematics Subject Classification (1985 Revision). Primary 58G99.

Partially supported by the NSF. 
Observe that each metric $e^{u_{t}} \cdot g, t \in \mathbb{R}$, is invariant under left translation by elements of $\Gamma$, and hence descends to a metric on $\Gamma \backslash F$.

Theorem A. The metrics $e^{u_{t}} \cdot g, t \in \mathbb{R}$, on $\Gamma \backslash F$ are isospectral but not isometric.

The example in Theorem A is based on a theorem of Sunada [S], which shows how to use finite group theory to construct isospectral manifolds. His construction was generalized by DeTurck and Gordon to infinite groups in the following way:

Theorem B [DG 2, Theorem 1.16]. Suppose that a Lie group $G$ acts by isometries on a Riemannian manifold $(M, g)$, so that $\Gamma_{1}$ and $\Gamma_{2}$ are cocompact discrete subgroups of $G$ which act freely and properly discontinuously on $M$, and that $\Gamma_{i} \backslash M$ is compact for $i=1,2$.

If for each $h \in G$, we have

$$
\begin{aligned}
& \sum_{\left[\gamma_{1}\right]_{\Gamma_{1}} \subset[h]_{G}} \operatorname{Vol}\left(C\left(\gamma_{1}, \Gamma_{1}\right) \backslash C\left(\gamma_{1}, G\right)\right) \\
= & \sum_{\left[\gamma_{2}\right]_{\Gamma_{2}} \subset[h]_{G}} \operatorname{Vol}\left(C\left(\gamma_{2}, \Gamma_{2}\right) \backslash C\left(\gamma_{2}, G\right)\right),
\end{aligned}
$$

then $\left(\Gamma_{1} \backslash M, g\right)$ is isospectral to $\left(\Gamma_{2} \backslash M, g\right)$.

Here, $[\gamma]_{H}$ denotes the conjugacy class of $\gamma$ in the group $H$, and $C(\gamma, H)$ denotes the centralizer of $\gamma$ in $H$. The volumes are computed relative to compatible Haar measures on the groups $C\left(\gamma_{i}, G\right)$; see [DG 2] for details.

Note that Sunada's theorem [S] corresponds to the special case $G$ a finite group.

In order to obtain isospectral conformally equivalent metrics, let $\left(M, g, G, \Gamma_{1}, \Gamma_{2}\right)$ satisfy the hypothesis of Theorem B. Suppose incidentally that there exists an element $\tau$ of Iso $(M, g) \backslash G$ such that $\Gamma_{2}=\tau \Gamma_{1} \tau^{-1}$. Then $\tau$ induces an isometry between $\left(\Gamma_{1} \backslash M, g\right)$ and $\left(\Gamma_{2} \backslash M, g\right)$.

Now let $u$ be a smooth function on $M$ which is $G$-invariant (i.e., $\Psi^{*} u=u \circ \Psi=u$ for all $\Psi \in G$ ) but not $\tau$-invariant. Observe that $\left(M, e^{u} g, G, \Gamma_{1}, \Gamma_{2}\right)$ also satisfies the hypothesis of Theorem 
B, so that

$$
\begin{aligned}
\operatorname{spec}\left(\Gamma_{1} \backslash M, e^{u} g\right) & =\operatorname{spec}\left(\Gamma_{2} \backslash M, e^{u} g\right) \\
& =\operatorname{spec}\left(\Gamma_{1} \backslash M, \tau^{*}\left(e^{u} g\right)\right) \\
& =\operatorname{spec}\left(\Gamma_{1} \backslash M, e^{u \circ \tau} g\right) .
\end{aligned}
$$

Hence $e^{u} g$ and $e^{u \circ \tau} g$ are isospectral conformally equivalent metrics.

To obtain Theorem A, we let $G$ be the subgroup of $F$ consisting of $\left\{f: x_{0} \in \mathbb{Z}\right\}$, and we let $\tau=\tau_{t}=f(t, 0,0,0,0,0,0)$, $M=F$.

We now turn to the question of whether $\left(\Gamma_{1} \backslash M, e^{u} g\right)$ and $\left(\Gamma_{1} \backslash M, e^{u \circ \tau} g\right)$ are isometric, in the case of Theorem A. To that end, we make use of a theorem of Milnor [Mi], which guarantees that any left-invariant metric on a nonabelian nilpotent Lie group has negative scalar curvature. Thus, $\left(\Gamma_{1} \backslash F, g\right)$ has constant negative scalar curvature. By a well-known lemma (see [BPY]), it follows that any conformal diffeomorphism $h$ of $\left(\Gamma_{1} \backslash F, g\right)$ is an isometry.

Now suppose that $h$ is a diffeomorphism of $\Gamma_{1} \backslash F$ which takes $e^{u} g$ to $e^{u \circ \tau} g . h$ is obviously a conformal diffeomorphism for the metric $g$, and so by the lemma must preserve $g$. It follows that $h$ is an isometry of the metric $\left(\Gamma_{1} \backslash F, g\right)$ which takes $u$ to $u \circ \tau$. But this last was shown to be impossible in the case at hand in [DG 2, Example 3.3 (ii)].

We remark that Brooks [B] and DeTurck and Gordon [DG 2, §3] showed under the same hypotheses on $M, g, G, \Gamma_{1}, \Gamma_{2}, \tau, u$ that the Schrödinger operators $\Delta+u$ and $\Delta+u \circ \tau$ on $\Gamma_{1} \backslash M$ are isospectral. Each of the examples of isospectral Schrödinger operators given in [B] and [DG 2] can be translated to the present setting to give examples of isospectral conformally equivalent metrics. Some other examples:

(i) Given any hyperbolic surface, or more generally any hyperbolic manifold $M$ whose fundamental group maps onto $\mathbb{Z} * \mathbb{Z}$, there is a finite covering $M^{\prime}$ of $M$ which admits a pair of isospectral conformally equivalent metrics $e^{u_{1}} g$ and $e^{u_{2}} g$ of negative curvature, where $g$ is the hyperbolic metric. Observe that $e^{u} g$ has negative curvature when $u$ is sufficiently close to the constant zero function.

This example arises from the construction in [B, §3]. 
(ii) There exist continuous families of isospectral, conformally equivalent metrics on $S^{3} \times S^{3} \times \mathbb{R}^{5} / \mathbb{Z}^{5}$. (The metrics are not conformally equivalent to the standard product metric.) To obtain these examples, define an action of the group $F$ of Theorem A on $S^{3} \times S^{3} \times \mathbb{R}^{5} / \mathbb{Z}^{5}$ (compare Example 4.6 of [DG 1]) and then apply the construction of [DG 2, §3].

\section{REFERENCES}

[Ber] P. Bérard, Variétés Riemanniennes isospectrales non-isométriques, Seminaire Bourbaki no. 705 (March 1989).

[B] R. Brooks, On manifolds of negative curvature with isospectral potentials, Topology 26 (1987), 63-66.

[BT] R. Brooks and R. Tse, Isospectral surfaces of small genus, Nagoya Math. J. 107 (1987), 13-24.

[BPY] R. Brooks, P. Perry, and P. Yang, Isospectral sets of conformally equivalent metrics, Duke Math. J. 58 (1989), 131-150.

[DG1] D. DeTurck and C. Gordon, Isospectral deformations Part I: Riemannian structures on two-step nilspaces, Comm. Pure Appl. Math. 40 (1987), 367387.

[DG2] __ Isospectral deformations Part II: Trace formulas, metrics, and potentials, Comm. Pure Appl. Math. 42 (1989), 1067-1096.

[Mi] J. Milnor, Curvatures of left invariant metrics on Lie groups, Adv. in Math. 21 (1976), 293-329.

[S] T. Sunada, Riemannian coverings and isospectral manifolds, Ann. of Math. 121 (1985), 169-186.

Department of Mathematics, University of Southern California, Los ANGEles, CALIFORNIA 90089-1113 90024

Current address: Department of Mathematics, UCLA, Los Angeles, California

Department of Mathematics, University of Washington at St. Louis, ST. Louis, Missouri 63130 